\title{
Use of urea filter paper disc to detect urease activity in Enterobacteriaceae by multipoint replication techniques
}

\author{
D B Winter, S N McDermott
}

\begin{abstract}
A new method of detecting urease activity in Enterobacteriaceae was developed. An $8.5 \mathrm{~cm}$ filter paper disc impregnated with $20 \%$ urea and $0.5 \%$ bromocresol purple was placed on the surface of a glucose fermentation plate after inoculation with a multipoint replicator and overnight incubation. This method was compared with the commercially prepared Mast urea agar (Multipoint) and Fuscoe's Urea Plate Medium. A total of 240 routine isolates of Enterobacteriaceae were tested for urease activity using the three methods. Sixty five isolates were positive by the three methods while 33 isolates gave differing results. The urea disc method was more sensitive for detecting urease activity in isolates of Klebsiella species, Morganella morganii, and Yersinia enterocolitica. It also overcame the problem associated with the other two media of diffusion of alkali end products through the medium.
\end{abstract}

The detection of urease activity is an important characterisation test used to help identify Enterobacteriaceae. Many laboratories use the agar medium of Christensen to detect urease activity. ${ }^{1}$ Where large numbers of organisms are identified daily, biochemical identification is achieved in many laboratories using the multipoint replication technique. This method provides a rapid, economical, and reliable identification system. ${ }^{23}$

Methods for detecting urease activity using the multipoint inoculation technique have been developed. These include Mast Urea Agar (Multipoint) (Mast Product Description Sheet IDM 32), which is based on Christensen's formulation but which has been modified slightly for use with the multipoint inoculation technique and the Urea Plate Medium of Fuscoe. ${ }^{4}$ Both these plate media rely on alkalinisation of the medium occurring when urease converts urea to ammonia. This $\mathrm{pH}$ change is readily detected using appropriate $\mathrm{pH}$ indicators.

We describe an alternative method for detecting urease activity in Enterobacteriaceae using multipoint inoculation techniques. This method uses a glucose fermentation plate inoculated with organisms to be tested, which, after overnight incubation, is overlayed with a filter paper disc impregnated with urea/ bromocresol. A blue spot appears on the filter paper over the colonies which produce the urease enzyme.

\section{Methods \\ IDENTIFICATION OF CLINICAL ISOLATES}

Two hundred and forty routine clinical isolates from urine specimens, wounds, and faeces were identified using the multipoint inoculation technique and were tested for tryptophan, methyl red, urease, $\mathrm{H}_{2} \mathrm{~S}$, citrate, malonate, DNAse, lysine, ornithine, arginine, adonitol, cellibiose, glucose, sucrose, mannitol, sorbitol, arabinose, raffinose, rhamnose, melibiose, xylose, lactose, indol, oxidase, oxidation/fermentation and motility. The number and identification of the 240 isolates is shown in table 1 .

FILTER PAPER UREASE DETECTION DISC

Nine $\mathrm{cm}$ in diameter, filter paper discs (Qualitative 1 Whatman, Maidstone, England) were trimmed to fit $8.5 \mathrm{~cm}$ diameter Petri dishes. These discs were saturated in an aqueous solution containing $20 \%$ urea and $0.05 \%$ bromocresol purple $\mathrm{w} / \mathrm{v}$ and laid on household greaseproof paper to dry in a fan air circulated incubator at $37^{\circ} \mathrm{C}$. After drying, the discs were stored in Petri dishes at room temperature before use.

\section{$1 \%$ GLUCOSE PLATE}

This comprises the following: $1 \mathrm{ml}$ phenol red 0.2\%; Oxoid Agar No 3 (L13) 1.7 g; Lab Lemco Oxoid (L29) 0.3 g; Tryptose Oxoid (L47) $0.5 \mathrm{~g}$; distilled water $90 \mathrm{ml} ; 10 \%$ glucose $10 \mathrm{ml}$; pour five plates per $100 \mathrm{ml}$ of agar. The formulation used is a non-inhibitory electrolyte deficient medium similar to those used in urine investigations, with the lactose replaced by glucose and with phenol red as the $\mathrm{pH}$ indicator.

After inoculation of the glucose fermenta-

Table 1 Routine isolates used to evaluate three methods for detecting urease activity

\begin{tabular}{lc}
\hline Organism & No of organisms identified \\
\hline Ecoli & 122 \\
Proteus mirabilis & 40 \\
Klebsiella pneumoniae & 27 \\
Enterobacter species & 15 \\
Klebsiella oxytoca & 9 \\
Citrobacter diversus & 8 \\
Serratia marcescens & 7 \\
Morganella morganii & 5 \\
Proteus vulgaris & 3 \\
Salmonella species & 2 \\
Yersinia enterocolitica & 2 \\
Total & 240 \\
\hline
\end{tabular}

\author{
S N McDermott \\ Correspondence to: \\ Dr D B Winter \\ Accepted for publication \\ 1 March 1990 \\ Division of Clinical \\ Institute of Med \\ Science, Frome Road, \\ Adelaide, South
}


Table 2 Isolates showing differences in urease activity among three methods

\begin{tabular}{|c|c|c|c|c|}
\hline \multirow[b]{2}{*}{ Organism } & \multirow[b]{2}{*}{ No of isolates } & \multicolumn{3}{|c|}{ Urease result } \\
\hline & & Urea disc & Mast agar & Urea plate method \\
\hline $\begin{array}{l}\text { Klebsiella oxytoca } \\
\text { Klebsiella pneumoniae } \\
\text { Enterobacter species } \\
\text { Morganella morgani } \\
\text { Morganella morgani } \\
\text { Yersinia enterocolitica } \\
\text { Proteus mirabilis } \\
\text { Citrobacter diversus } \\
\text { Citrobacter diversus } \\
\text { Enterobacter species }\end{array}$ & $\begin{array}{l}6 \\
8 \\
4 \\
3 \\
3 \\
2 \\
2 \\
3 \\
3 \\
2\end{array}$ & $\begin{array}{l}+ \\
+ \\
+ \\
+ \\
+ \\
+ \\
+ \\
- \\
- \\
-\end{array}$ & $\begin{array}{l}- \\
- \\
- \\
- \\
- \\
- \\
+ \\
+ \\
+ \\
+\end{array}$ & $\begin{array}{l}+ \\
+ \\
+ \\
- \\
- \\
- \\
+ \\
+ \\
+\end{array}$ \\
\hline
\end{tabular}

tion plate using a 32 pronged replicating device the plate was incubated at $36^{\circ} \mathrm{C}$ for 18 hours. The results of glucose utilisation were recorded. Any organism which did not produce acid from glucose was excluded from the urease detection test. The impregnated filter paper disc was then laid on the surface of the plate and a cotton wool swab was used to remove air bubbles by pressing it down on the plate. The whole disc became yellow due to acid present in the medium produced by glucose breakdown. Within five minutes those growths which produced urease caused a purple spot to develop on the filter paper due to the release of alkaline endproducts which made the $\mathrm{pH}$ indicator change from yellow to purple. The filter paper remained yellow in colour above growths where urease was not produced.

\section{Results}

Of the 240 isolates, 90 were positive using the urea filter paper disc, 75 using the Mast urea agar, and 88 using the urea plate medium. One hundred and forty two isolates were negative for urease enzyme activity by all three methods. Sixty five isolates were positive by the three methods, while thirtythree isolates gave differing results. The isolates which gave differing results among the three methods are shown in table 2 .

\section{Discussion}

The urea filter paper circle disc was developed because existing media seemed to lack sensitivity in detecting urease activity in certain organisms while the diffusion of alkaline endproducts of strong urease producers obscured the test results of other organisms.

The Mast urea agar (Multipoint) method was slightly modified from Christensen's formulation for use with the multipoint inoculation technique. The concentration of urea was reduced and the agar concentration raised in an attempt to prevent colour spread from one growth spot to the next.

We found that the colour spread from the strong urease producers, such as Proteus mirabilis, was still quite extensive, especially if several of these isolates were present on the same plate and in close proximity. The spread of the colour reaction made the urease result of neighbouring colony spots very difficult to interpret. The relatively poor sensitivity of conventional multipoint urea medium was highlighted by finding that a large proportion of Klebsiella species (14 of 36) and Morganella morganii (three of five) failed to show urease activity in the Mast urea agar. Those Klebsiella species which did give a positive urease result on the Mast urea agar resulted in a change that was generally weak.

Fuscoe's urea plate medium was specifically developed for the multipoint inoculation technique and was recommended for the demonstration of urease activity in up to nine bacterial strains on a single $9 \mathrm{~cm}$ plate rather than using a 32 pronged replicator. It was also observed in the urea plate medium that the large amounts of alkali produced by strong urease producing organisms would diffuse through the medium and obscure the results of neighbouring colony spots. The results of urease detection for the Klebsiella species were superior using the urea plate medium compared with the Mast urea agar, but like the Mast agar, the results for Morganella morganii were negative in three of the five isolates. Neither of these two media detected urease activity in the two isolates of Yersinia enterocolitica after 18 hours of incubation.

The urea disc method relies on several important considerations. Firstly, urease is a constitutive enzyme and is synthesised by certain bacteria regardless of the presence or absence of its substrate urea. ${ }^{5}$ Therefore, organisms capable of producing the urease enzyme will do so on the glucose plate even though the medium is devoid of urea. Secondly, by definition, all Enterobacteriaceae are glucose fermenters and glucose is a stimulator of urease activity, particularly in those organisms which hydrolyse urea slowly. The energy provided by glucose fermentation causes the increased stimulation of the urease enzyme by increasing the rate of metabolism and cell reproduction. ${ }^{5}$ Thirdly, the concentration of glucose in the glucose fermentation is $1 \%$. This is the recommended concentration for carbohydrate fermentation tests because it reduces the possibility of alkaline reversion occurring. ${ }^{5}$ Because the urease test relies on the demonstration of alkalinity, a $1 \%$ concentration of glucose will prevent false positive reactions occurring due to alkaline reversion (acetoin production). The urea disc method for detecting urease activity was found to overcome the interpretation problems of alkaline diffusion if the results were read within 30 minutes of the disc being placed on the agar plate. The colour intensity for the positive reactions was fully developed 
within five minutes and contained in the area of the growth spot for up to 30 minutes. The urea disc detected urease activity in strains of Klebsiella, Proteus, Morganella morganii and Yersinia enterocolitica. The urea disc method was, however, less sensitive in detecting urease activity in some strains of Enterobacter species and Citrobacter diversus than the other two methods.

We believe that when incubated at $36^{\circ} \mathrm{C}$ these organisms were unable to produce sufficient urease enzyme and consequently sufficient alkali endproducts to overcome the acid endproducts that resulted from glucose fermentation.

According to Topley and Wilson, ${ }^{6}$ many Enterobacter species give atypical biochemical reactions unless tested at lower temperatures, perhaps indicating increased biochemical activity at lower temperatures. Further testing comparing urease production of organisms at $36^{\circ} \mathrm{C}$ and $30^{\circ} \mathrm{C}$ using the urea filter disc method showed isolates of both Enterobacter and Citrobacter species which gave positive reactions at $30^{\circ} \mathrm{C}$ but not at $36^{\circ} \mathrm{C}$. Isolates of Yersinia enterocolitica gave more intense positive reactions at $30^{\circ} \mathrm{C}$ while the results for other organisms were unaffected when incu- bated at $30^{\circ} \mathrm{C}$. We therefore recommend that the urea filter paper disc method for detecting urease activity be done at $30^{\circ} \mathrm{C}$.

The urea disc method for detecting urease activity in Enterobacteriaceae using the multipoint inoculation technique was easy to perform and interpret. The discs can be made up in single batches and stored at room temperature with no loss of activity. The sensitivity of the method facilitates identification of some organisms, especially Klebsiella, Morganella, and Yersinia, and it overcomes the problem of alkaline diffusion associated with other urease detection media used in multipoint replicate techniques.

1 Christensen WB. Urea decomposition as a means of differentiating Proteus and paracolon culture from each other and from Salmonella and Shigella types. J Bacteriol 1946; 52:461-6.

2 Pease AA. Biochemical identification of the Enterobacteriaceae using a multipoint inoculation system. Med Lab Sci 1983;40:349-53.

3 Pease AA. Antimicrobial susceptibility testing and biochemical identification using multipoint inoculation: 5 years experience. Med Lab Sci 1988;45:28-33.

4 Fuscoe FJ. A multiple inoculation urea plate medium. Med Lab Technol 1974;31:247-52.

Lab Technol 1974;31:247-52.
5 MacFaddin JF. Biochemical tests for identification of medical bacteria. 2nd Ed. Baltimore: Williams and Wilkins, 1980. 6 Wilson G, Miles A. Topley and Wilson's principles of bacteriology, virology and immunity. Seventh Edn. Vol 2. Systematic bacteriology. Butler and Tanner Ltd, 1985. 